\title{
LOCALIZAÇÃO DE FRATURAS EM ROCHAS GRANÍTICAS, NO MUNICÍPIO DE CAPÃO BONITO-SP, BRASIL
}

\author{
Abad Oliveira Souza Júnior' \\ Jorge Luís Porsani² \\ Recebido em 6 de mai., 2002 / Aceito em 22 de nov., 2003 \\ Received May 6, 2002 / Accepted Nov. 22, 2003
}

\section{ABSTRACT}

Ground Penetrating Radar - GPR and Resistivity methods were used with the objective to detect fracture zones and the top of fresh rock, in ornamental granite mine, located near Capão Bonito City, São Paulo State, Brazil. Eight profiles of reflections GPR and 19 WARR's Soundings were acquired using antennas of 25, 50, 100 and $200 \mathrm{MHz}$. Two vertical electrical sounding were acquired too. Analysis of GPR's profiles allowed to locate stronger dipping and sub horizontal reflectors, probably related with the fractures zones filled with water. Besides, were identified two shadow zones that can be related with intrusions of diabase's dike, and one hyperbolic reflector with a boulder. The fresh rock was identified about $23 \mathrm{~m}$ of depth. The results obtained probably will go help the project of mine engineering looking forward to advancing of work front.

Keywords: GPR-Ground Penetrating Radar, Electrical Resistivity, Fractures Zones, Ornamental Granite, Capão Bonito, São Paulo, Brazil.

\section{RESUMO}

Os métodos GPR-Ground Penetrating Radar e Resistividade Elétrica foram utilizados com o objetivo de localizar zonas de fraturas e o topo da rocha sã, numa pedreira de granito ornamental, localizada próxima à cidade de Capão Bonito, Estado de São Paulo-Brasil. Oito perfis de reflexão GPR e 19 sondagens de velocidade WARR-Wide Angle Reflection and Refraction foram adquiridas utilizando-se as antenas de 25, 50, 100 e $200 \mathrm{MHz}$. Também foram adquiridas duas SEV's-Sondagens Elétricas Verticais. As análises dos perfis GPR permitiram localizar fortes refletores inclinados e subhorizontais, provavelmente relacionados com zonas de fraturas preenchidas com água. Além disso, foram identificadas duas zonas sem reflexões rasas que podem estar relacion adas com intrusões de diques de diabásio, e um refletor hiperbólico relacionado com um matacão. A rocha sã foi identificada a $23 \mathrm{~m}$ de profundidade. Os resultados obtidos irão auxiliar o projeto da engenharia de minas orientando 0 avanço na frente de trabalho.

Palavras-chave. GPR-Ground Penetrating Radar, Granitos Ornamentais, Fraturas, Juntas de alívio, Capão Bonito.

\footnotetext{
Pós-Graduação em Geofísica no IAG/USP - Rua do Matão 1226 - Cidade Universitária - São Paulo - SP - CEP 05508-090 - Tel.: (11) 30914734 - Fax: (11) 30915034.

2 IAG/USP - Departamento de Geofísica - Rua do Matão 1226 - Cidade Universitária - São Paulo - SP - CEP 05508-090 - Tel.: (11) 30914734 - Fax: (11) 30915034. E-mail: porsani@iag.usp.br.
} 


\section{INTRODUÇÃOO}

Este trabalho tem como objetivos a caracterização do topo da rocha sã, a localização de zonas de fraturas e possíveis juntas de alívio, estruturas comuns em maciços graníiticos. Para alcançar tais objetivos foram empregados os métodos geofísicos: Ground Penetrating Radar GPR e eletrorresistividade. Os dados foram adquiridos numa pedreira de granito ornamental localizada no Município de Capão Bonito - SP, pertencente à empresa SOMIBRÁS (Sociedade Brasileira de Mineração $S / A)$. A caracterização geoelétrica dos materiais de subsuperfície é importante para se discriminar, a zona de alteração da rocha, o topo da rocha sã e a localização de zonas de fraturas e juntas de alívio. 0 imageamento em detalhe de subsuperfície permite subsidiar os projetos de engenharia de minas, por exemplo, a cubagem do volume de solo que deverá ser retirado (conhecido como bota fora), a localização de zonas mais favoráveis à exploração do granito ornamental e, conseqüentemente, na cubagem da jazida explorável. Dessa maneira, os custos relativos à explotação da jazida poderão ser minimizados. Os dados geofísicos adquiridos estão sendo interpretados de maneira integrada, sendo comparados com informações de dados geológicos de superfície. Isto permitirá obter um modelo geofísico que auxilie os projetos de engenharia de minas para a exploração da jazida.

\section{GEOLOGIA DA ÁREA DE ESTUDO}

A área de estudos localiza-se no município de Capão Bonito, distante cerca de 350km da cidade de São Paulo - SP (Figura 1). A região esta localizada na borda da Bacia Sedimentar do Paraná, correspondendo às porç̃oes sul do estado de São Paulo e leste do estado do Paraná (CHIODI FILHO et. al., 1983). Os dados geofíisicos foram adquiridos na pedreira de granito pertencente à empresa SOMIBRÁS, situada aproximadamente a 20 quilometros do município de Capão Bonito. A área em estudo é caracterizada principalmente por rochas graníticas e corpos granitóides, ocorrendo localmente diques de diabásio. 0 granito de Capão Bonito destaca-se por ser uma rocha de coloração avermelhada, com granulação grossa e isotrópica. Devido às suas características de alta homogeneidade textural e sua cor avermelhada, conferem ao granito um padrão estético apreciado internacionalmente, o que favoreceu 0 desenvolvimento de intensa lavra em todo o maciço, sendo exportado como rocha ornamental para a Itália e Japão, cujo

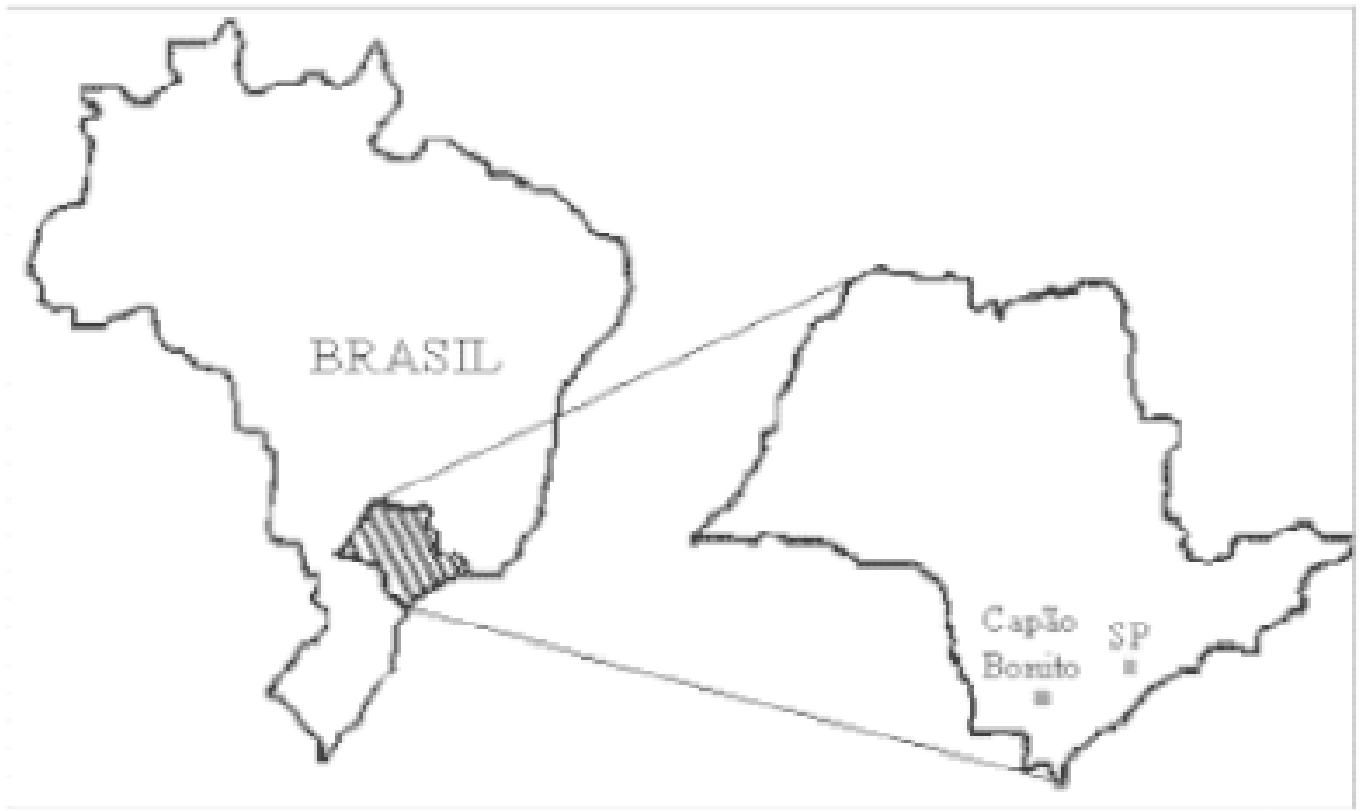

Figura 1 - Mapa de localização esquemático da área de estudos. Figure 1 - Location map from studies area. 
nome comercial é Granito Capão Bonito ou Rubi Red Granite. Conforme observações de campo, na área da pedreira os corpos graníiticos aparecem estruturados contendo zonas de fraturas, matacões e diques de diabásio, bem como a cabeceira do córrego dos Alves dentro da área da lavra.

\section{METODOLOGIA E AQUISIÇÃO DOS DADOS}

Neste trabalho foram utilizados os métodos geofísicos GPR e eletrorresistividade. 0 método GPR consiste em obter uma imagem de alta resolução da subsuperfície, através da transmissão de ondas eletromagnéticas em altas freqüências, por meio de uma antena transmissora colocada no terreno. As mudanças nas propriedades elétricas dos materiais de subsuperfície fazem com que parte do sinal retorne a superfície. As ondas de radar refletidas e difratadas em subsuperfície são captadas por meio de uma antena receptora, onde são registradas em função do tempo duplo, que é amplificada, digitalizada e gravada no disco rígido de um notebook (PORSANI, 1999).

Os levantamentos geofísicos foram realizados ao longo de estradas de acesso a pedreira e na frente de lavra. Para a aquisição dos dados GPR foi utilizado o equipamento RAMAC/GPR da empresa sueca - MALA - Geoscience, pertencente ao Departamento de Geofísica do IAG/USP. Foram adquiridos oito perfis de reflexão GPR, com a técnica de afastamento constante, utilizando-se antenas de 25,50, 100 e 200 MHz, visando obter uma boa composição de sinais eletromagnéticos. Nesse caso, sobre o mesmo perfil, consegue-se obter uma boa penetração da onda com as antenas de $25 \mathrm{MHz}$ e uma boa resolução com as antenas de 200MHz. Os perfis de reflexão tiveram como objetivos a localização de zonas de fraturas, juntas de alívio e imagear o topo da rocha sã. Os dados foram adquiridos em três locais distintos, totalizando cerca de 693m de perfis GPR.

Sobre os perfis de GPR foram adquiridas 19 sondagens de velocidade do tipo WARR - Wide Angle Refraction and Reflection, utilizando-se antenas com frequênncia central 50, 100 e 200MHz. A análise das sondagens de velocidade pelo método Semblance permitiu calcular uma velocidade de propagação da onda EM em subsuperfície de $0,1 \mathrm{~m} / \mathrm{ns}$, que é compatível com rochas graníticas (PORSANI, 1999). A Figura 2 mostra uma sondagem do tipo WARR obtida com as antenas de $100 \mathrm{MHz}$.

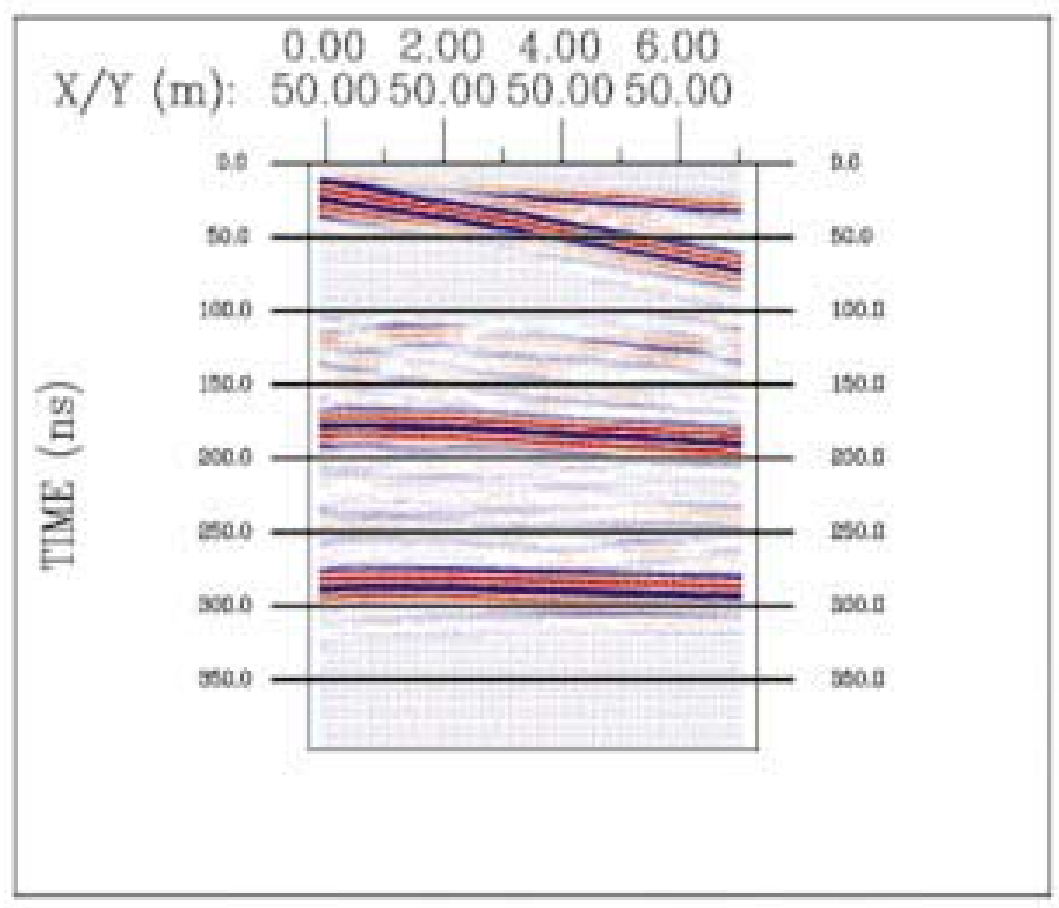

Figura 2 - Sondagem WARR adquirida com as antenas de $100 \mathrm{MHz}$.

Figure 2 - WARR souding acquired by $100 \mathrm{MHz}$ antennas. 
Neste trabalho ser̃̃o apresentados somente dois perfis de reflexão com afastamento constante. A Figura 3 mostra um perfil GPR de 102m, obtido com antenas de 50MHz. Este perfil foi adquirido ao longo de uma estrada, na parte mais alta da pedreira, onde o manto de alteração é mais espesso. Próximo ao final desse perfil encontra-se uma nascente do córrego Alves.

A Figura 4 mostra um perfil GPR de 62m, obtido com antenas de 100MHz. Esse perfil foi adquirido na frente de lavra localizada na parte mais baixa da pedreira, onde aflora a rocha sã. Naquele local 0 granito está bastante fraturado. Conforme observaçōes de campo, alguns planos de fraturas apresentavam umidade.

0 outro método geofísico utilizado foi o da eletrorresistividade, que emprega uma corrente elétrica artificial, introduzida no terreno através de dois eletrodos $A$ e B, com o objetivo de medir o potencial gerado em outros dois eletrodos $\mathrm{M} \mathrm{e} \mathrm{N} \mathrm{(ELIS,} \mathrm{1999).} 0$ equipamento utilizado foi um resistivímetro francês marca lris, modelo Syscal R2. Foram realizadas duas sondagens elétricas verticais com arranio Schlumberger, AB/2 de 150m de extensão, sobre um perfil de GPR obtido ao longo da estrada.

\section{PROCESSAMENTO PRELIMINAR DOS DADOS GEOFÍSICOS}

Os dados GPR estão sendo atualmente processados com 0 soffware GRADIX da Interpex. As principais etapas utilizadas no processamento básico dos dados foram: a) correção do wow, isto é, ruído em baixa freqüência, b) correção do tempo zero, c) aplicação de ganhos no tempo (ganho linear, esférico, exponencial e programado), d) aplicação de filtro passa banda, e) conversão de tempo/profundidade.

A SEV está sendo modelada através do soffware de inversão 10 RESIXIP da Interpex, e não será apresentada neste trabalho.

\section{DISCUSSÃO DOS RESULTADOS}

A sondagem de velocidade do tipo WARR (Figura 2) está localizada na posiçã̃o de $74 \mathrm{~m}$ do perfil GPR de 50MHz. Nota-se a existência de dois refletores hiperbólicos, um em torno de 160ns e 0 outro em torno de $280 \mathrm{~ns}$. Na conversão tempo/profundidade $(v=0,1 \mathrm{~m} /$ ns) do perfil de reflexão correspondente, esses refletores relacionam-se com alvos geológicos em 8 e $14 \mathrm{~m}$ de profundidade, respectivamente (Figura 3).

Os perfis de reflexão GPR mostram imagens nítidas de refletores inclinados, subhorizontais e hiperbólicos. No perfil GPR de 102m (Figura 3), alguns refletores anômalos merecem destaque. Nos primeiros $5 \mathrm{~m}$ de profundidade, os refletores irregulares correspondem ao capeamento do granito alterado. Entre 3 e $11 \mathrm{~m}$ observa-se uma ausência de reflexão, denominada zona de sombra. Esta região corresponde à intrusão de um dique de diabásio que aflora e corta o perfil GPR, conforme constatado no campo. Entre 85 e 95m, e até $5 \mathrm{~m}$ de profundidade, observa-se a presença de um refletor inclinado, que é um indicativo da presença de fratura. De 5 a $10 \mathrm{~m}$ de profundidade, os refletores inclinados entre 30 e 45m, e entre 80 e $95 \mathrm{~m}$ são indicativos de fraturas. Segundo a estatigrafia da rocha graníitca no local investigado (CHIODI FILHO et al., 1983), esse trecho corresponde à rocha granítica alterada. Em torno de 10 e $13 \mathrm{~m}$ de profundidade ocorrem dois fortes refletores subhorizontais, que podem estar associados com o contato entre a rocha mais alterada e a rocha graníitica menos alterada, respectivamente. Este contato é favorecido por fraturas horizontais, estruturas comuns em granitos e que na área de lavra estão preenchidas de água, provavelmente devido à ocorrência de uma nascente do córrego Alves, distante cerca de $100 \mathrm{~m}$ do final do perfil. Esse fato pode explicar os fortes refletores subhorizontais em torno de 10 e $13 \mathrm{~m}$ de profundidade. Nota-se também um nítido refletor hiperbólico com vértice na posição de $52 \mathrm{~m}$ e em torno de $10 \mathrm{~m}$ de profundidade. Este refletor está relacionado com a presença de um matacão em subsuperfície (a velocidade de ajuste para a hipérbole é de $0,1 \mathrm{~m} / \mathrm{ns}) .0$ refletor que ocorre em torno de $13 \mathrm{~m}$ de profundidade apresenta uma descontinuidade entre as posições de 60 e 62,5m. Essa característica é semelhante à zona de sombra que ocorre entre 3 e $11 \mathrm{~m}$ desse perfil, e portanto, pode ser associada com a presença de um segundo dique de diabásio que não aflora na superfície. 0 refletor que está em torno de $23 \mathrm{~m}$ de profundidade e entre 65 e 90m, também pode estar relacionado com a presença de fraturas subhorizontais preenchidas com água e demarca, provavelmente, o topo da rocha sã. Nota-se que a partir dessa profundidade não ocorrem mais refletores, indicando que a rocha é mais homogênea e sem fraturas.

0 perfil de reflexão de $62 \mathrm{~m}$ realizado na frente de lavra, com antenas de $100 \mathrm{MHz}$ (Figura 4), é caracterizado por vários refletores inclinados, variando desde a superfície até cerca de $15 \mathrm{~m}$ de profundidade. Observa-se também um forte refletor subhorizontal em torno de $14 \mathrm{~m}$ de profundidade. É provável que estes refletores estejam relacionados com a presença de fraturas, inclinadas e subhorizontais, preenchidas de água.

\section{CONCLUSÕES}

0 método GPR permitiu localizar zonas de fraturas inclinadas e subhorizontais, até cerca de $25 \mathrm{~m}$ de profundidade. Essas fraturas provavelmente estão preenchidas de água. Foi localizado um matacão 


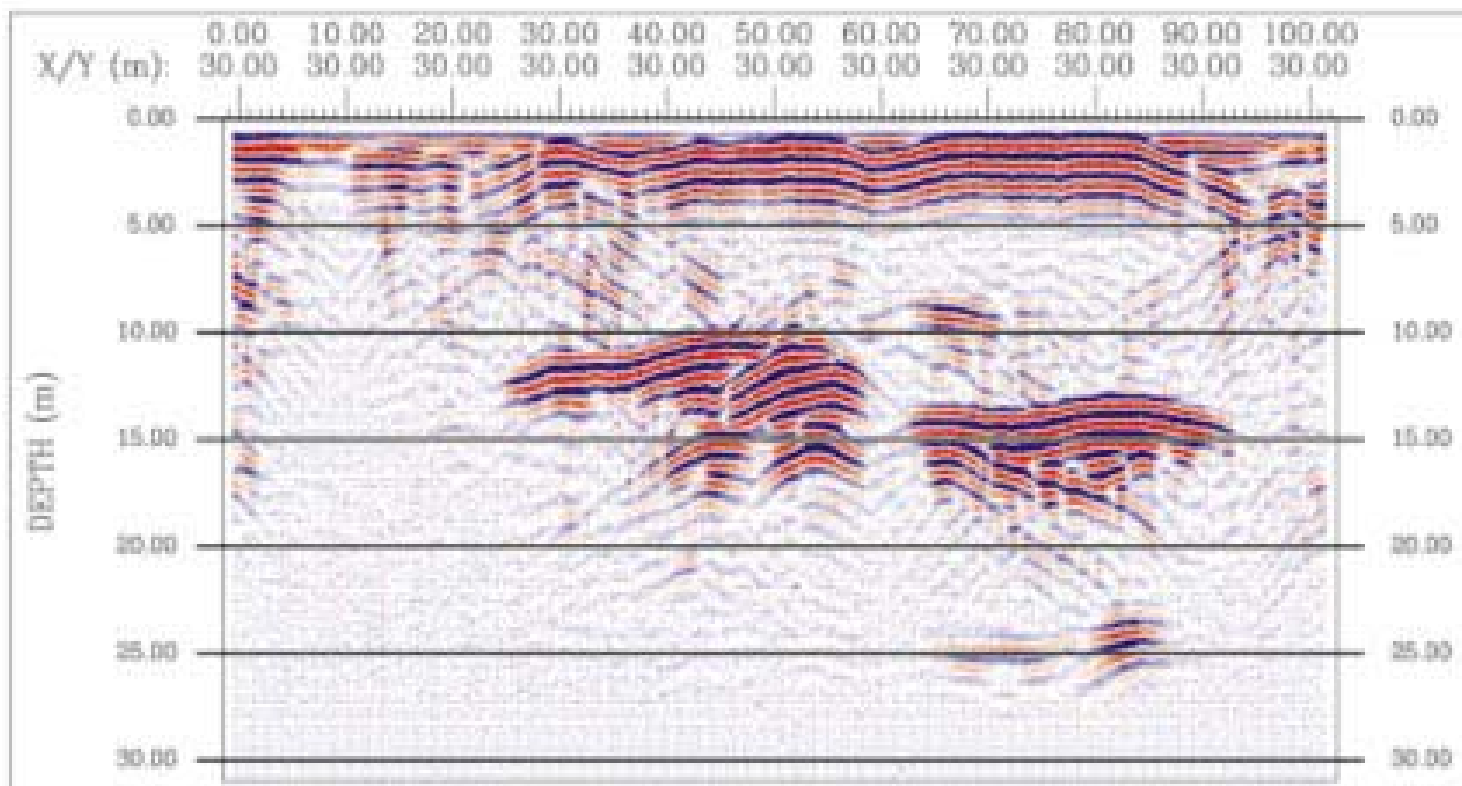

Figura 3 -. Perfil GPR no alto da pedreira com as antenas de $50 \mathrm{MHz}$ Figure 3 - GPR profile in the top of quarry by $50 \mathrm{MHz}$ antennas.

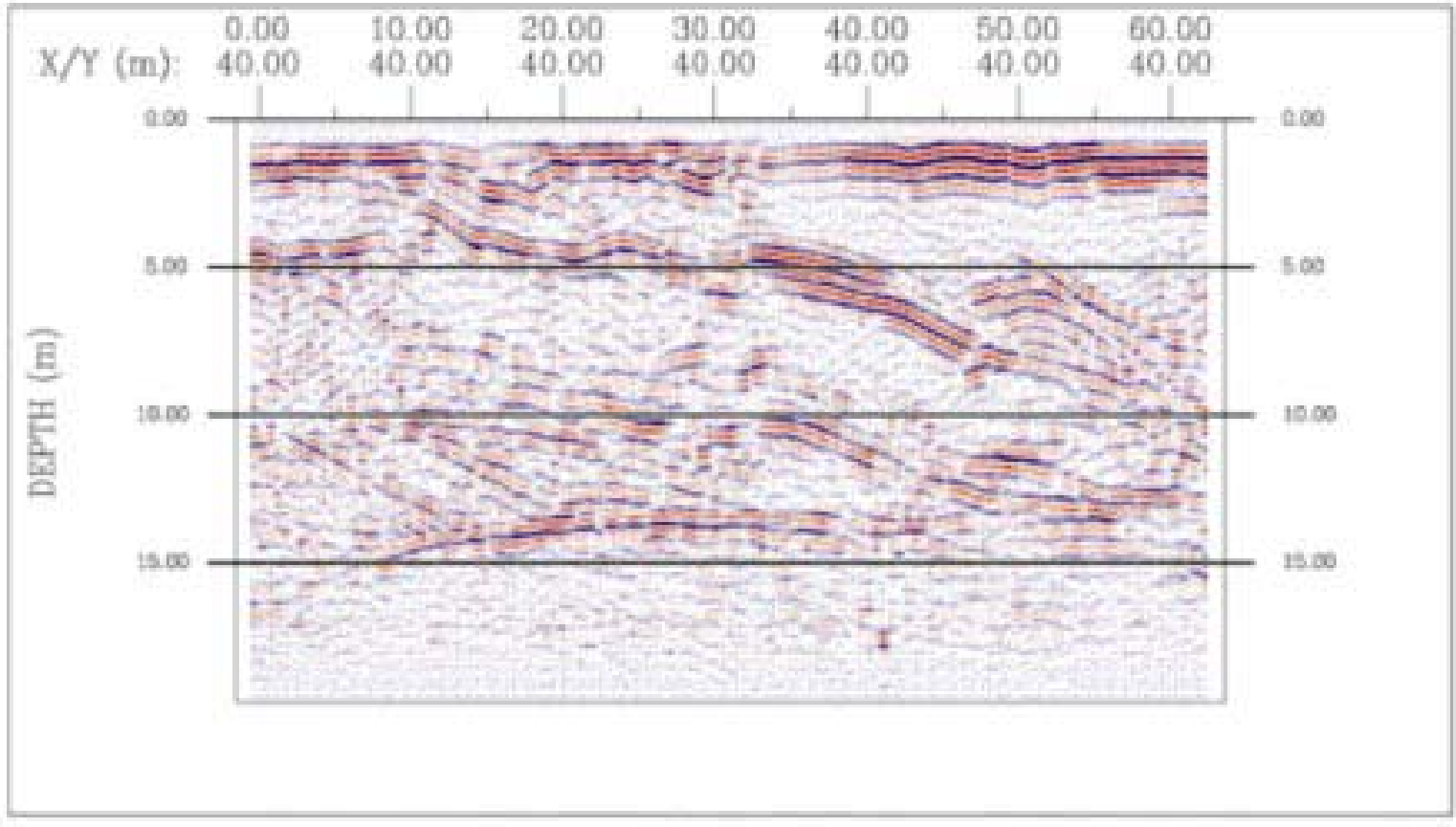

Figura 4 - Perfil GPR na frente de lavra com as antenas de 100MHz. Figure 4-GPR profile in exploitation front by $100 \mathrm{MHz}$ antennas. 
em torno de $10 \mathrm{~m}$ de profundidade, bem como a intrusão de um dique de diabásio, confirmado pelo reconhecimento de campo. Um segundo dique de diabásio foi inferido em torno de $13 \mathrm{~m}$ de profundidade não aflorando na área pesquisada. 0 topo da rocha sã foi localizado em torno de $23 \mathrm{~m}$ de profundidade. As informações obtidas da interpretação dos perfis GPR estão de acordo com as informações geológicas de superfície. Este trabalho fornece informações importantes ao engenheiro de minas, permitindo um melhor planejamento quanto à exploração da jazida, no sentido de calcular o volume de rocha a ser explotável (granitos sem fraturas) e ainda, direcionar 0 avanço da frente de lavra.

\section{Agradecimentos}

Ao CNPq, pela concessão da bolsa de mestrado. Ao Departamento de Geofísica do IAG/USP, pela infra-estrutura. Ao Prof. Dr. Jorge Luís Porsani, pela orientação desta pesquisa e proveitosas discussões. Ao Professor Francisco Yukio Hiodo e ao colega Jorge Eduardo da Silva, pela ajuda na aquisição de dados. Ao pesquisador Marcelo Filipov, pela discussão sobre a geologia da área.

\section{REFERÊNCIAS}

CHIODI FILHO, C. et. al. Projeto Capão Bonito. São Paulo, out. 1983. Relatório final, CPRM. v. 1.

ELIS, V. R. Avaliação da aplicabilidade de métodos elétricos de prospecção geofísica no estudo de áreas utilizadas para disposição de resíduo. 1999. 264p. Tese (Doutorado)-Instituto de Geociências e Ciências Exatas, Universidade Estadual Paulista, Rio Claro, 1999.

PORSANI, J. L. Ground Penetrating Radar (GPR): proposta metodológica de emprego em estudos geológico-geotécnicos nas regiões de Rio Claro e Descalvado - SP. 1999. 145 p. Tese (Doutorado)-Instituto de Geociências e Ciências Exatas, Universidade Estadual Paulista, Rio Claro, 1999.

\section{NOTAS SOBRE OS AUTORES}

Abad Oliveira Souza Júnior é engenheiro mecânico formado pela UNESP, campus de Guaratinguetá-SP e Tecnólogo em Construção Civil pela FATEC-SP. Mestrado em Geofísica pelo IAG-USP em 2002, desenvolvendo a pesquisa intitulada: "Investigações Geofísicas na Pedreira de Granito Ornamental, no Municípío de Capão Bonito-SP, utilizando-se os Métodos GPR e Eletrorresistividade". Atualmente trabal ha como Engenheiro na empresa SPB Engenharia.

Jorge Luís Porsani é geólogo pelo Instituto de Geociências da UFBa (1987). Mestrado em Geofísica pelo Núcleo de Pesquisas Geofísicas Aplicadas à Prospecção de Hidrocarbonetos da UFPa (1991). De 1991 a 1996, trabalhou como Geofísico no Centro de Pesquisas da PETROBRAS. Doutorado em Geociências e Meio Ambiente pelo Instituto de Geociências e Ciências Exatas da UNESP (1999). Desde 1/12/ 1998 é docente do departamento de Geofísica do IAG/USP, atuando com métodos geoelétricos aplicados à geologia. geotecnia, meio ambiente e arqueologia. Atualmente, é Vice Coordenador do Programa de Pós-Graduação em Geofísica e Coordenador do Projeto de Instalação do Sítio Controlado de Geofísica Rasa do IAG (Fapesp 02/07509-1). 